\title{
GCU
}

Glasgow Caledonian

University

University for the Common Good

\section{Deep residual neural network for EMI event classification using bispectrum representation}

Mitiche, Imene; Jenkins, Mark David; Boreham, Philip ; Nesbitt, Alan; Stewart, Brian G.;

Morison, Gordon

Published in:

2018 26th European Signal Processing Conference (EUSIPCO)

DOI:

10.23919/EUSIPCO.2018.8553177

Publication date:

2018

Document Version

Author accepted manuscript

Link to publication in ResearchOnline

Citation for published version (Harvard):

Mitiche, I, Jenkins, MD, Boreham, P, Nesbitt, A, Stewart, BG \& Morison, G 2018, Deep residual neural network for EMI event classification using bispectrum representation. in 2018 26th European Signal Processing Conference (EUSIPCO). IEEE, pp. 186-190, 2018 26th European Signal Processing Conference, Rome, Italy, 3/09/18. https://doi.org/10.23919/EUSIPCO.2018.8553177

\section{General rights}

Copyright and moral rights for the publications made accessible in the public portal are retained by the authors and/or other copyright owners and it is a condition of accessing publications that users recognise and abide by the legal requirements associated with these rights.

Take down policy

If you believe that this document breaches copyright please view our takedown policy at https://edshare.gcu.ac.uk/id/eprint/5179 for details of how to contact us. 


\title{
Deep Residual Neural Network for EMI Event Classification Using Bispectrum Representations
}

\author{
Imene Mitiche*, Mark David Jenkins*, Philip Boreham $\ddagger$ \\ Alan Nesbitt*, Brian G. Stewart ${ }^{\dagger}$ and Gordon Morison* \\ * School of Engineering and Built Environment \\ Glasgow Caledonian University, \\ Glasgow, United Kingdom \\ $\dagger$ Institute of Energy and Environment \\ University of Strathclyde, \\ Glasgow, United Kingdom \\ $\ddagger$ Innovation Centre for Online Systems \\ Bere Regis, \\ United Kingdom
}

\begin{abstract}
This paper presents a novel method for condition monitoring of High Voltage (HV) power plant equipment through analysis of discharge signals. These discharge signals are measured using the Electromagnetic Interference (EMI) method and processed using third order Higher-Order Statistics (HOS) to obtain a Bispectrum representation. By mapping the time-domain signal to a Bispectrum image representations the problem can be approached as an image classification task. This allows for the novel application of a Deep Residual Neural Network (ResNet) to the classification of HV discharge signals. The network is trained on signals into 9 classes and achieves high classification accuracy in each category, improving upon our previous work on this task.
\end{abstract}

\section{INTRODUCTION}

Electromagnetic Interference (EMI) measurement is a method utilised for Partial Discharge (PD) detection in HighVoltage (HV) systems [1]. PD is an electrical discharge that occurs due to failure in an insulation medium [2], which if neglected, could cause equipment breakdown resulting high maintenance or replacement costs. EMI diagnosis involves EMI signals measurement followed by experts analysis in order to identify presence of faults or insulation degradation in the HV equipment including cables, generators, motors, transformers etc. [3]. This technique captures PD as well as other discharge sources such as Corona, Arcing etc. The current EMI diagnosis method is manual, this means larger time spent on data acquisition and analysis is unavoidable, which implies high remuneration. Furthermore, this method is not practical for continuous monitoring. There may also be a lack in trained and experienced EMI experts and interpretation of EMI data could be challenging [4].

A proposed solution in this paper is to develop an automatic fault diagnosis model based on state-of-the-art signal processing techniques. A literature review on PD detection and classification techniques is provided in [5]-[7]. The summary of these papers includes a comparison of PD measurement methods and covers a large number of feature extraction and classification techniques that were proposed for PD identifi- cation. The current literature lacks studies on classification of EMI discharge sources, yet it has been widely exploited for PD measurement since 1980 to the present [1]. Authors in [8] and [9] attempted for the first time feature extraction and machine learning application to EMI signals in order to develop an EMI intelligent system based on experts system. The idea was to classify different EMI discharge sources, using relevant features and Multi-Class Support Vector Machine (MCSVM). Classification accuracy results demonstrated a successful performance with room for improvement.This work presents an extension to previous EMI classification with the aim to classify a larger EMI data set that contains more variety in discharge sources collected at additional HV operating sites.

The approach is to employ Deep Learning technique with relevant features. HOS analysis, specifically Bispectrum, has recently received the attention of biomedical [10]-[12] and audio [13], [14] applications as a feature extraction technique. The authors in [10] utilised Bispectrum entropies to retrieve information from lung sound signals with the aim of identifying lung health related condition (normal, asthma or pneumonia). This feature extraction method was introduced in [11] and [12] to machine learning and neural network classifiers, on electrocardiogram signals for classification of five different types of cardiac arrhythmia and emotion recognition purposes, respectively. Mel frequency Cepstral coefficients and HOS features were extracted in [13] and [14] from non-linear and non-stationary music audio signals, with the aim of musical instruments and instrument family classification, using different neural networks (e.g., counter propagation, feed forward neural network). Bispectrum analysis was also exploited on vibration signals, with non-stationarity characteristics, to identify gearbox fault types [15]. The previously cited papers demonstrated the ability of Bispectrum to retrieve robust features for the analysis of complex, non-linear and non-stationary signals. This is the main motivation to employ Bispectrum, as EMI signals are believed to be of similar nature. The motivation for using deep learning is to improve classification performance. 
This paper is structured as follows. Section 2 defines the feature extraction and classification algorithms employed in this work. Section 3 introduces the experimental set-up with a brief description on EMI data collection. Section 4 presents the classification results in terms of classification accuracy and confusion matrices. Finally, Section 5 draws conclusions from this research with future plans.

\section{FEATURE EXTRACTION AND ClASSIFICATION ALGORITHMS DESCRIPTION}

\section{A. Bispectrum Analysis}

In time-domain HOS analysis, the first-order moment of a discrete signal $x[n] ; n=1, \ldots, N$ is the mean $\mu$ and its secondorder moment is the variance $\sigma^{2}$, both are defined in Equation 1 and 2 respectively.

$$
\begin{gathered}
\mu=E(x[n]) . \\
\sigma^{2}=E\left(x[n] x\left[n+\tau_{1}\right]\right) .
\end{gathered}
$$

The third-order moment of the signal is defined as:

$$
\gamma=E\left(x[n] x\left[n+\tau_{1}\right] x\left[n+\tau_{2}\right]\right) .
$$

where $\tau_{1}$ and $\tau_{1}$ are time lags. Assuming that the signal $x[n]$ is zero mean, then the second- and third- order cumulants are equal to the respective moments [16]. In frequency-domain definition, the third-order cumulant spectrum, known as the Bispectrum, is the Fourier Transform (FT) of the third-order moment which can be defined as:

$$
\begin{array}{r}
B\left(f_{1}, f_{2}\right)=\sum_{\tau_{1}=1}^{N} \sum_{\tau_{2}=1}^{N} \sum_{n=1}^{N} x[n] x\left[n+\tau_{1}\right] x\left[n+\tau_{2}\right] \\
\cdot e^{-j 2 \pi \cdot\left(f_{1} \tau_{1}+f_{2} \tau_{2}\right)} .
\end{array}
$$

Let $n+\tau_{1}=m$ and $n+\tau_{2}=k$, by rewriting Equation 4 and splitting the exponent, Bispectrum can be reformulated as:

$$
\begin{gathered}
B\left(f_{1}, f_{2}\right)=\left\{\sum_{m=1}^{N} x[m] e^{-j 2 \pi f_{1} m}\right\} \cdot\left\{\sum_{k=1}^{N} x[k] e^{-j 2 \pi f_{2} k}\right\} \\
\cdot\left\{\sum_{n=1}^{N} x[n] e^{+j 2 \pi\left(f_{1}+f_{2}\right) n}\right\}=X\left(f_{1}\right) X\left(f_{2}\right) X^{*}\left(f_{1}+f_{2}\right) .
\end{gathered}
$$

where $X(f)$ is the FT and $X^{*}(f)$ is the complex conjugate of $X(f)$. The Bispectrum is a complex measure that contains both magnitude and phase information, unlike the power spectrum. The Bispectrum reveals its content in two independent frequencies $f_{1}$ and $f_{2}$. Figure 1 shows an example Bispectrum contour map of PD and E signals. The time domain of the two signals is provided in Figure 2. It is observed that both time signals have similar content in terms of pulses which may be challenging for their classification. However, the Bispectrum of these two signals reveals a unique signature for each signal type.

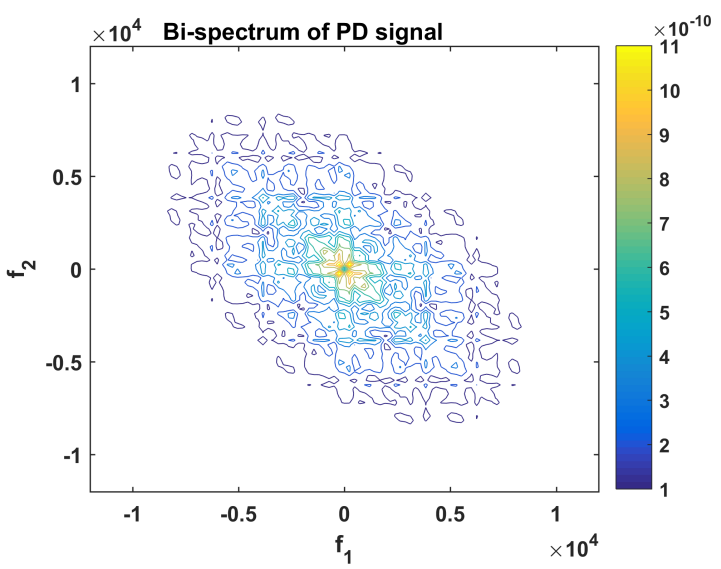

(a) $\mathrm{PD}$

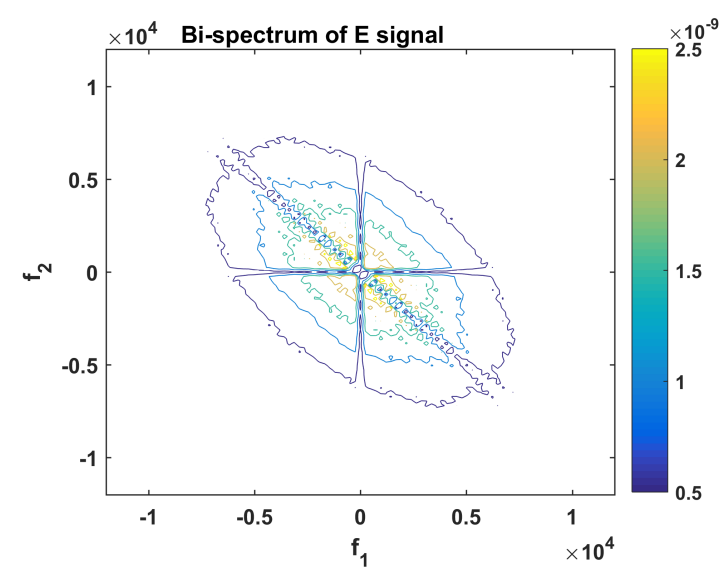

(b) $\mathrm{E}$

Fig. 1. Bispectrum image of PD and E events

\section{B. Deep Residual Network}

The application of Deep Residual Networks (ResNets) [17] to the image classification problem has become very popular since the use of a very deep networks to achieve state-of-theart results on challenges such as ImageNet [18]. A residual network differs from a standard Convolutional Neural Network (CNN) through the implementation of identity skip connections or shortcuts. These shortcut connections have been shown to reduce the potential for vanishing/exploding gradients [19] and reduce training loss and error [17]. To exploit the power of ResNet architectures in image classification, this work attempts to use the image representation of the Bispectrum of the time domain signal for classification. The residual network utilised in this work is based on the 34 layer ResNet from [17]. Figure 3 shows two possible configurations of what will be referred to as residual blocks. These blocks consist of a combination of convolutions, Batch Normalisations (BN) and ReLU Activations [20] as well as a shortcut connection with associated addition. These residual blocks are used to build the complete ResNet-34 architecture as detailed in Table I.

The standard ResNet architecture utilises the residual blocks depicted in Figure 3a. Later work presented in [21] extends 
TABLE I

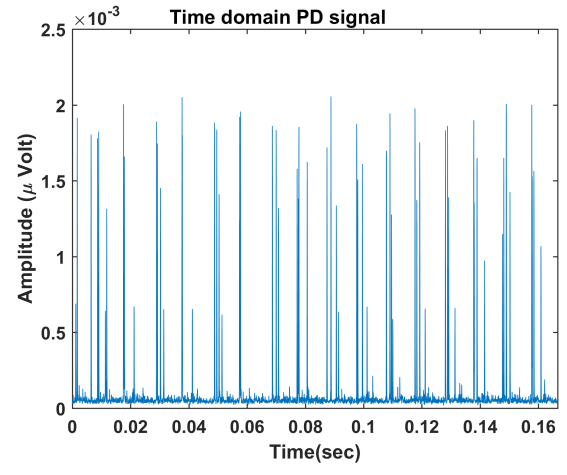

(a) PD time-series

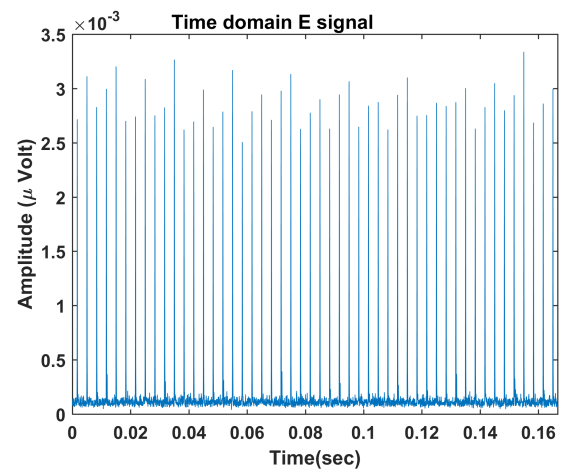

(b) E time-series

Fig. 2. Time-domain signal of PD and E events

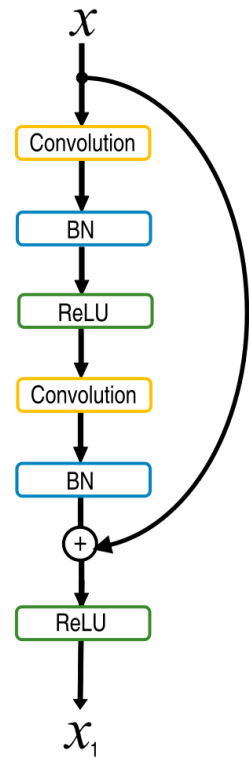

(a) Standard Configuration

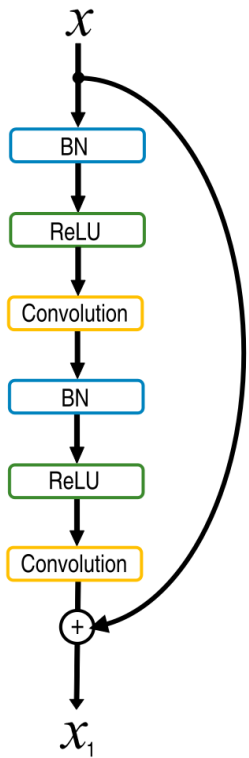

(b) Pre-Activation
Fig. 3. Residual Block Configurations
STRUCTURE OF THE RESNET-34 ARCHITECTURE WHERE A RESIDUAL BLOCK CAN BE SUBSTITUTED FOR ANY SUITABLE BLOCK CONFIGURATION SUCH AS THOSE IN FIGURE 3

\begin{tabular}{c|c|c|c|c} 
Layer & Kernel & Depth & Stride & Operation \\
\hline \hline 1 & $7 \times 7$ & 64 & 2 & Conv \\
\hline 2 & $3 \times 3$ & - & 1 & MaxPool \\
\hline 3 & $3 \times 3$ & 64 & 1 & Residual Block x 3 \\
\hline 4 & $3 \times 3$ & 128 & 1 & Residual Block x 4 \\
\hline 5 & $3 \times 3$ & 256 & 1 & Residual Block x 6 \\
\hline 6 & $3 \times 3$ & 512 & 1 & Residual Block x 3 \\
\hline 7 & \multicolumn{5}{|c}{ Average Pool, Fully Connected, SoftMax } \\
\hline
\end{tabular}

upon the original sturcture of the residual blocks by utilising a pre-activation configuration. By applying the BN and ReLU functions before the convolutions, the pre-activation residual block structure (shown in Figure 3b) has been shown to reduce test error as well as prevent overtraining of the model. This is the residual block structure implemented in this work with learning rate of 0.01 .

\section{EMI DATA ACQUISITION AND EXPERIMENTAL SET-UP}

EMI measurement follows the method specified in the CISPR 16 (Committee International Special des Perturbations Radioelectriques) Standard [22], which involves a quasi-peak detector with various filter bandwidths over a frequency span. Examples include the B filter which operates over $150 \mathrm{kHz}-$ $30 \mathrm{MHz}$, with a $6 \mathrm{~dB}$ bandwidth of $9 \mathrm{kHz}$, and the $\mathrm{C}$ filter from $30-300 \mathrm{MHz}$ with a $6 \mathrm{~dB}$ bandwidth of $120 \mathrm{kHz}$. The quasipeak detector has a sampling time of 1 second per selected frequency and it is fast enough to capture the discharges intensity [23]. Further details on EMI measured frequency spectrum can be found in [3], [23], [24]. EMI time resolved signals can be retrieved from the frequencies of interest in the spectrum. The signals are subsequently analysed by EMI experts with the aim of identifying the presence of any discharge source [25], based on past accumulated experience of fault diagnosis and forensic confirmation. The signals in this paper were measured during the assets' operation by means of High Frequency Current Transformer (HFCT) which is connected around the neutral earth cable of the asset. The signals are recorded at a sampling rate of $24 \mathrm{kHz}$ on a PD Surveyor 200 (PDS200) instrument which operates following the CISPR16 standard for EMI filter types. The PDS200 is able to detect and analyse Radio Frequency Interference (RFI) in addition to EMI radiations in the lower frequency range [50kHz-1GHz].

The studied signals in this paper were collected and measured, using EMI method, from different apparatus (generator, isolated-phase bus, steam turbine generator, transformer etc.) across eleven power sites. Figure 5 illustrates an example scenario of EMI signals measured from neutral earth connection of a generator, at one of the studied sites. The obtained time signals were converted to audio then analysed by EMI experts who subsequently identify and label the discharge types present in the apparatus. Findings indicated the presence 


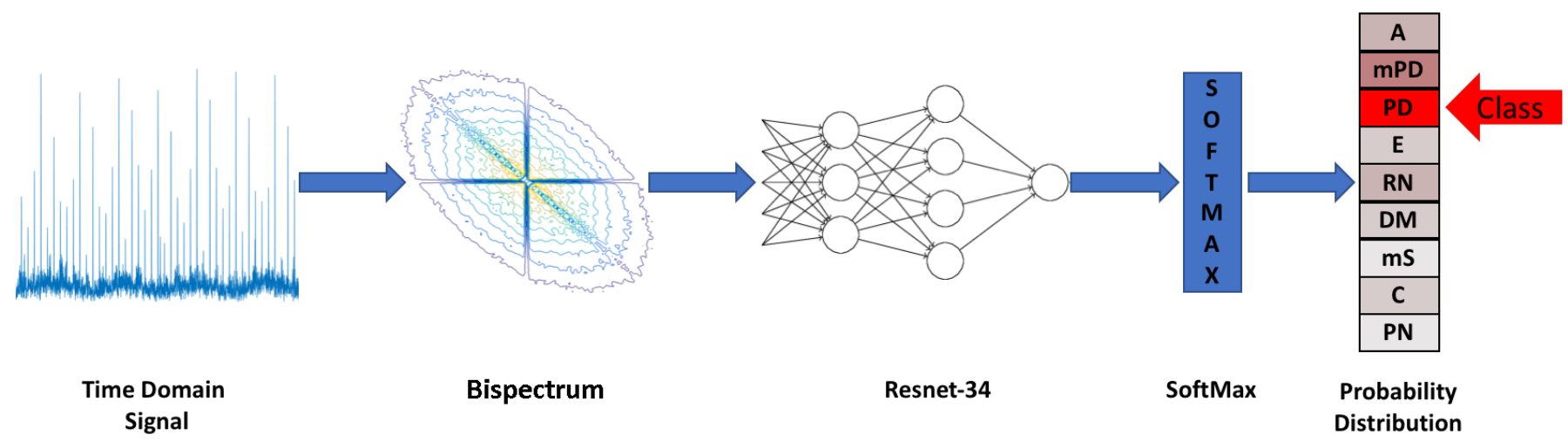

Fig. 4. Time series Classification approach using Bi-Spectrum image feature and ResNet-34 Neural Network

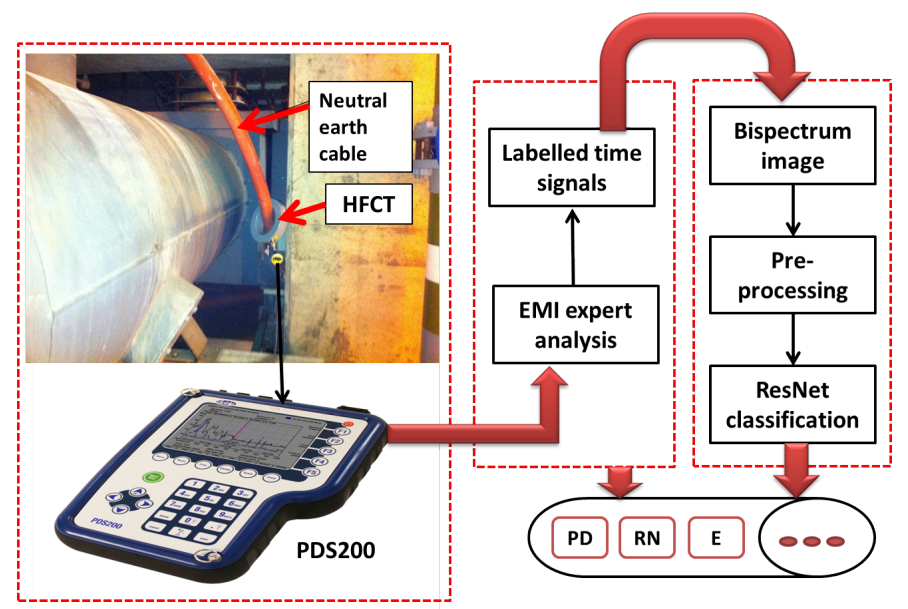

Fig. 5. Overall experimental set-up, from EMI data measurement, EMI expert analysis and labelling, to the proposed classification approach

of 9 events (classes) known as Arcing (A), Corona (C), Data Modulation (DM), Exciter (E), PD, Process Noise (PN), Random Noise (RN), minor PD (mPD) and micro Sparking $(\mathrm{mS})$. The second stage involves the application of signal processing techniques including feature extraction and the ResNet-34 to the measured and labelled EMI signals. Here, the magnitude of the Bispectrum is extracted from each signal to obtain relevant information. Pre-processing such as image scaling is also performed in order to aid the learning process. The network is trained on 14,000, validated on 2000 and tested on 2000 BiSpectrum images from 9 classes. The model is implemented using Keras and Tensorflow and training takes approximately one hour on an NVIDIA Titan Xp GPU with 12GB RAM.

\section{ClassificAtion Results}

Experiment results are presented in terms of classification accuracy as well as confusion matrix, since 9 classes are presented to the developed model. Table II presents results from each run output of a 10 validation folds and the average performance. It is observed that the highest classification ac-
TABLE II

ClASSIFICATION ACCURACY RESULTS

\begin{tabular}{c|c} 
Run No. & Acc. \% \\
\hline \hline 1 & $\underline{97.23}$ \\
2 & 95.10 \\
3 & $\underline{97.23}$ \\
4 & 89.82 \\
5 & 86.24 \\
6 & 93.33 \\
7 & 89.14 \\
8 & 92.81 \\
9 & 85.55 \\
10 & 95.97 \\
\hline Average acc. \% & $\mathbf{9 2 . 2 4}$
\end{tabular}

curacy was achieved in the first and third runs with $97.23 \%$, as underlined in the table. Whereas, the lowest accuracy obtained is $85.55 \%$. Yet, it has been found that the overall performance is consistent and the average accuracy surpasses the $90 \%$ score which is excellent for a classification of 9 different classes. In order to better understand the obtained performance results, the average confusion matrix is illustrated in Figure 6. The major misclassification is seen between $\mathrm{mPD}$ and PD with $17.46 \%$ of mPD being classified as PD. This could be because $\mathrm{MPD}$ is a subset fault type of PD. In fact mPD represents the occurrence of PD at lower repetition rate and discharge level which makes their classification ambiguous.

\section{CONCLUSION}

In this work an application of Deep ResNets was proposed on EMI real world signals for pattern recognition of different discharge sources that represent potential faults (e.g., PD, A) or common events (e.g., PN, RN). The approach exploits image classification techniques by using the Bispectrum image of EMI signals in a 34-layer ResNet model. Performance consistency of the developed model was demonstrated based on the average total accuracy of all classes and runs and the prediction accuracy for each individual class in the averaged runs. On the basis of the promising findings presented in this paper, a second stage analysis to distinguish between PD and $\mathrm{mPD}$ is of interest for future work. 


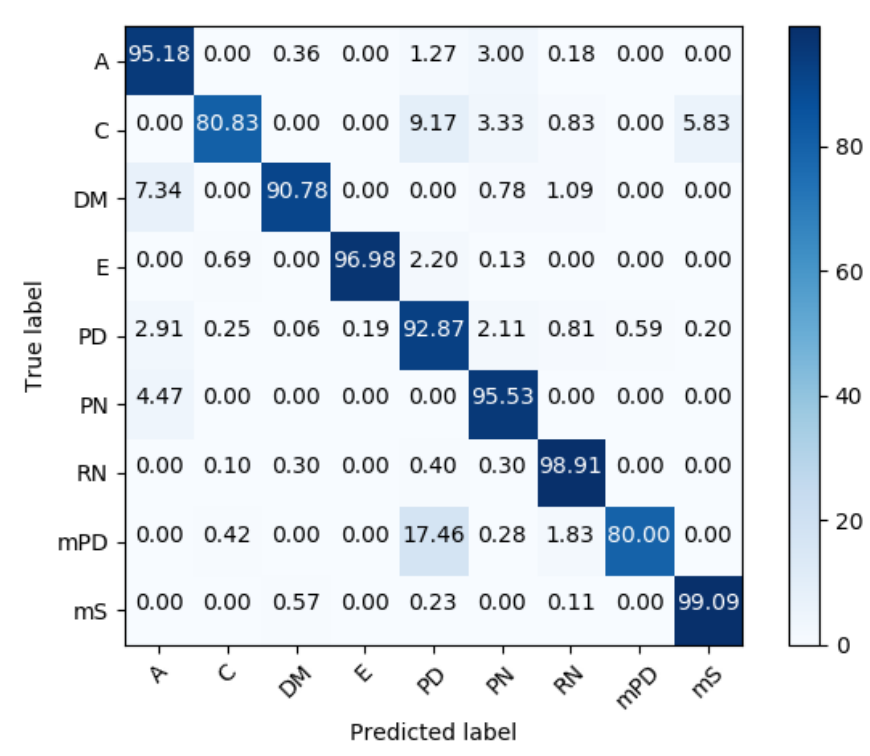

Fig. 6. Average Confusion Matrix calculated over the 10 validation folds

\section{REFERENCES}

[1] J. E. Timperley, D. Buchanan, and J. M. Vallejo, "Electric generation condition assessment with electromagnetic interference analysis," IEEE Transactions on Industry Applications, vol. PP, pp. 1-1, 2017.

[2] F. Kreuger, Industrial High Voltage: 4. Coordinating, 5. Testing, 6. Measuring. Delft University Press, 1992.

[3] J. E. Timperley and J. M. Vallejo, "Condition assessment of electrical apparatus with emi diagnostics," IEEE Transactions on Industry Applications, vol. 53, pp. 693-699, 2017.

[4] C. V. Maughan, "Electromagnetic interference (emi) testing of electrical equipment," in 2011 Electrical Insulation Conference (EIC)., 2011, pp. 340-344.

[5] W. J. K. Raymond, H. A. Illias, A. H. A. Bakar, and H. Mokhlis, "Partial discharge classifications: Review of recent progress," Measurement, vol. 68, pp. $164-181,2015$.

[6] M. Wu, H. Cao, J. Cao, H. L. Nguyen, J. B. Gomes, and S. P. Krishnaswamy, "An overview of state-of-the-art partial discharge analysis techniques for condition monitoring," IEEE Electrical Insulation Magazine, vol. 31, pp. 22-35, 2015.

[7] M. Mondal and G. Kumbhar, "Detection, measurement, and classification of partial discharge in a power transformer: Methods, trends, and future research," IETE Technical Review, vol. 0, pp. 1-11, 2017.

[8] I. Mitiche, G. Morison, A. Nesbitt, P. Boreham, and B. G. Stewart, "Classification of partial discharge emi conditions using permutation entropy-based features," in 2017 25th European Signal Processing Conference (EUSIPCO), 2017, pp. 1375-1379.

[9] I. Mitiche, G. Morison, A. Nesbitt, M. Hughes-Narborough, P. Boreham, and B. G. Stewart, "Classification of partial discharge signals by combining adaptive local iterative filtering and entropy features," in 2017 IEEE Conference on Electrical Insulation and Dielectric Phenomena., 2017, pp. 335-338.

[10] S. Li and Y. Liu, "Feature extraction of lung sounds based on bispectrum analysis," in 2010 Third International Symposium on Information Processing, 2010, pp. 393-397.

[11] A. Lanat, G. Valenza, C. Mancuso, and E. Scilingo, "Robust multiple cardiac arrhythmia detection through bispectrum analysis," Expert Systems with Applications, vol. 38, pp. 6798 - 6804, 2011.

[12] N. Kumar, K. Khaund, and S. M. Hazarika, "Bispectral analysis of eeg for emotion recognition," Procedia Computer Science, vol. 84, pp. $31-35,2016$, proceeding of the Seventh International Conference on Intelligent Human Computer Interaction (IHCI 2015).

[13] D. Bhalke, C. R. Rao, and D. Bormane, "Hybridisation of mel frequency cepstral coefficient and higher order spectral features for musical instru- ments classification," Archives of Acoustics, vol. 41, pp. 427 - 436, 2016.

[14] D. G. Bhalke, C. B. R. Rao, and D. S. Bormane, "Musical instrument classification using higher order spectra," in 2014 International Conference on Signal Processing and Integrated Networks (SPIN), 2014, pp. $40-45$.

[15] F. A. H. Jinying, S. B. P. Hongxia, and T. C. B. Shihua, "Bispectrum entropy feature extraction and its application for fault diagnosis of gearbox," in International Conference on Fuzzy Systems, 2010, pp. 1-6.

[16] K. C. Chua, V. Chandran, U. R. Acharya, and C. M. Lim, "Application of higher order statistics/spectra in biomedical signalsa review," Medical Engineering and Physics, vol. 32, pp. 679 - 689, 2010.

[17] K. He, X. Zhang, S. Ren, and J. Sun, "Deep residual learning for image recognition," in Proceedings of the IEEE conference on computer vision and pattern recognition, 2016, pp. 770-778.

[18] O. Russakovsky, J. Deng, H. Su, J. Krause, S. Satheesh, S. Ma, Z. Huang, A. Karpathy, A. Khosla, M. Bernstein et al., "Imagenet large scale visual recognition challenge," International Journal of Computer Vision, vol. 115, pp. 211-252, 2015.

[19] C. Szegedy, W. Liu, Y. Jia, P. Sermanet, S. Reed, D. Anguelov, D. Erhan, V. Vanhoucke, A. Rabinovich et al., "Going deeper with convolutions," in 2015 IEEE Conference on Computer Vision and Pattern Recognition (CVPR), 2015, pp. 1-9.

[20] V. Nair and G. E. Hinton, "Rectified linear units improve restricted boltzmann machines," in Proceedings of the 27th international conference on machine learning (ICML-10), 2010, pp. 807-814.

[21] K. He, X. Zhang, S. Ren, and J. Sun, "Identity mappings in deep residual networks," in European Conference on Computer Vision. Springer, 2016, pp. 630-645.

[22] CISPR/CIS/A - Radio-interference measurements and statistical methods, EMC 33.100.10 - Emission, 2015.

[23] J. E. Timperley, "Comparison of pda and emi diagnostic measurements [for machine insulation]," in Conference Record of the the 2002 IEEE International Symposium on Electrical Insulation (Cat. No.02CH37316), 2002, pp. 575-578.

[24] J. E. Timperley, J. M. Vallejo, and A. Nesbitt, "Trending of emi data over years and overnight," in 2014 IEEE Electrical Insulation Conference (EIC), Philadelphia, USA, 2014, pp. 176-179.

[25] J. E. Timperley, "Audio spectrum analysis of emi patterns," in 2007 Electrical Insulation Conference and Electrical Manufacturing Expo, Nashville, USA, 2007, pp. 39-41.

[26] — "Detection of insulation deterioration through electrical spectrum analysis," in 1983 EIC 6th Electrical/Electronical Insulation Conference, 1983, pp. 60-64. 